\title{
16S metagenomic microbial composition analysis of kefir grain using MEGAN and BaseSpace
}

\begin{abstract}
Kefir is a unique cultured product traditionally made from the fermentation of milk with kefir grains. Metagenomics analysis of kefir grain is essential to understand the composition of microbial populations in the kefir grain. Many microbial populations have been reported in kefir grains from different parts of the world. Although the kefir from Malaysian kefir grain is regularly consumed locally, no report has been made on the kefir grain microbial profile. The present study used kefir grain obtained locally and the microbial composition in the kefir grain was determined using Next Generation Sequencing (NGS). The taxonomic results analysis obtained when using BaseSpace (Illumina) and MEGAN were compared. The software agreed that Lactobacillus genus dominated the samples and the predominant species was L. kefiranofaciens (81.45 $191.93 \%)$ while L. kefiri $(2.01$ ï 2.47\%) was the second in abundance. The results suggested that Malaysian kefir grain contained the same top two predominant species using both software methods and the microbial composition between both software did not vary significantly.
\end{abstract}

Keyword: Kefir; Lactobacillus kefiranofaciens; Lactobacillus kefiri 